\title{
Exponential decay in a spin bath
}

\author{
W. A. Coish, ${ }^{1,2}$ Jan Fischer, ${ }^{2}$ and Daniel Loss $^{2}$ \\ ${ }^{1}$ Institute for Quantum Computing and Department of Physics and Astronomy, \\ University of Waterloo, 200 University Ave. W., Waterloo, ON, N2L 3G1, Canada \\ ${ }^{2}$ Department of Physics, University of Basel, Klingelbergstrasse 82, 4056 Basel, Switzerland
}

(Dated: October 23, 2018)

\begin{abstract}
We show that the coherence of an electron spin interacting with a bath of nuclear spins can exhibit a well-defined purely exponential decay for special ('narrowed') bath initial conditions in the presence of a strong applied magnetic field. This is in contrast to the typical case, where spin-bath dynamics have been investigated in the non-Markovian limit, giving super-exponential or power-law decay of correlation functions. We calculate the relevant decoherence time $T_{2}$ explicitly for free-induction decay and find a simple expression with dependence on bath polarization, magnetic field, the shape of the electron wave function, dimensionality, total nuclear spin $I$, and isotopic concentration for experimentally relevant heteronuclear spin systems.

PACS numbers: 03.65.Yz, 72.25.Rb, 31.30.Gs
\end{abstract}

\section{INTRODUCTION}

There are many proposals to use the spin states of electrons in confined structures for coherent manipulation, leading to applications in quantum information processing and ultimately, quantum computation. $\frac{1,2,3.4,5}{\mathrm{~A} \text { se- }}$ ries of recent experiments on such spin states in quantum dots,$\frac{6,7}{=}$ electrons bound to phosphorus donors in silicon,,$\frac{8}{2}$ NV centers in diamond, $, 4,9,10$ and molecular magnets 11,12 have shown that the hyperfine interaction between confined electron spins and nuclear spins in the surrounding material is the major obstacle to maintaining coherence in these systems.

Previous studies of this decoherence mechanism have pointed to the non-Markovian nature of a slow nuclearspin environment, leading to non-exponential coherence decay $13,14,15,16,17,18,19,20,21,22,23,24,25$ These results suggest that it may be necessary to revise quantum error correction protocols to accommodate such a 'nonstandard', but ubiquitous environment. $\underline{26}$ In the present work, we show that virtual flip-flops between electron and nuclear spins can lead to a well-defined Markovian dynamics, giving simple exponential decay in a large Zeeman field and for particular initial conditions (a 'narrowed' $\stackrel{27}{2}$ nuclearspin state). Moreover, we calculate the decoherence time $T_{2}$, revealing the dependence on many external parameters for a general system.

The rest of this paper is organized as follows: In Sec. III we introduce the Hamiltonian for the Fermi contact hyperfine interaction and derive an effective Hamiltonian for electron spin dynamics which is valid in a strong magnetic field. In Sec. III we present the Markov approximation and its range of validity, giving an analytical expression for the decoherence time $T_{2}$. We also give bounds for the non-Markovian corrections to our expression. Sec. IV gives a discussion of the decoherence rate for a homonuclear system and in Sec. $\mathrm{V}$ we generalize these results for a heteronuclear spin bath, providing explicit analytical expressions for $T_{2}$ within our Born-Markov approximation. We conclude in Sec. VI and present additional technical details in Appendices $\mathrm{A}, \mathrm{D}$

\section{HAMILTONIAN}

We begin from the Hamiltonian for the Fermi contact hyperfine interaction between a localized spin- $1 / 2 \mathbf{S}$ and an environment of nuclear spins,

$$
H_{\mathrm{hf}}=b S^{z}+b \sum_{k} \gamma_{k} I_{k}^{z}+\mathbf{S} \cdot \mathbf{h} ; \quad \mathbf{h}=\sum_{k} A_{k} \mathbf{I}_{k} .
$$

Here, $\mathbf{I}_{k}$ is the nuclear spin operator for the spin at site $k$ with associated hyperfine coupling constant $A_{k}$, $b=g^{*} \mu_{\mathrm{B}} B$ is the electron Zeeman splitting in an applied magnetic field $B$ and $\gamma_{k}$ is the nuclear gyromagnetic ratio in units of the electron gyromagnetic ratio (we set $\hbar=1): \gamma_{k}=g_{I_{k}} \mu_{N} / g^{*} \mu_{B}$. For an electron with envelope wave function $\psi(\mathbf{r})$, we have $A_{k}=v_{0} A^{i_{k}}\left|\psi\left(\mathbf{r}_{k}\right)\right|^{2}$, where $A^{i_{k}}$ is the total coupling constant to a nuclear spin of species $i_{k}$ at site $k$ and $v_{0}$ is the volume of a unit cell containing one nucleus. For convenience, we define $A=\sqrt{\sum_{i} \nu_{i}\left(A^{i}\right)^{2}}$, where $\nu_{i}$ is the relative concentration of isotope $i$. The envelope function $\psi(\mathbf{r})$ of the bound electron has finite extent, and consequently there will be a finite number $\sim N$ of nuclei with appreciable $A_{k}$. For typical quantum dots, $N \sim 10^{4}-10^{6}$, and for donor impurities or molecular magnets, $N \sim 10^{2}-10^{3}$. In Eq. (11) we have neglected the anisotropic hyperfine interaction, dipole-dipole interaction between nuclear spins, and nuclear quadrupolar splitting, which may be present for nuclear spin $I>1 / 2$. The anisotropic hyperfine interaction gives a small correction for electrons in a primarily $s$-type conduction band $\stackrel{28}{,}$ such as in III-V semiconductors or Si. Nuclear dipole-dipole coupling can give rise to dynamics in the spin bath, which can lead to electronspin decay due to spectral diffusion on a time scale found to be $T_{M} \sim 10-100 \mu \mathrm{s}$ for GaAs quantum dots $\frac{14,29,30}{1,2}$ These times are one to two orders of magnitude longer 
than the $T_{2}$ we predict for a GaAs quantum dot carrying $N=10^{5}$ nuclei (see Fig. 3, below). For smaller systems, we expect the decay mechanism discussed here to dominate dipole-dipole effects substantially. The quadrupolar splitting has also been measured for nanostructures in GaAs, giving inverse coupling strengths on the order of $100 \mu \mathrm{s}, \frac{31}{4}$ comparable to the dipole-dipole coupling strength, so quadrupolar effects should become relevant on comparable time scales.

For large $b$, we divide $H_{\mathrm{hf}}=H_{0}+V_{\mathrm{ff}}$ into an unperturbed part $H_{0}$ that preserves $S^{z}$ and a term $V_{\mathrm{ff}}=$ $\frac{1}{2}\left(S_{+} h_{-}+S_{-} h_{+}\right)$that leads to energy non-conserving flip-flops between electron and nuclear spins. $\frac{15}{\underline{15}}$ We eliminate $V_{\mathrm{ff}}$ to leading order by performing a SchriefferWolff-like transformation: $\bar{H}=e^{S} H_{\mathrm{hf}} e^{-S} \approx H=$ $H_{0}+\frac{1}{2}\left[S, V_{\mathrm{ff}}\right]$, where $S=\frac{1}{\mathrm{~L}_{0}} V_{\mathrm{ff}}$, and $\mathrm{L}_{0}$ is the unperturbed Liouvillian, defined by $\mathrm{L}_{0} O=\left[H_{0}, O\right]$. The resulting effective Hamiltonian is of the form 29,32 (see Appendix $B$ )

$$
H=(\omega+X) S^{z}+D
$$

The operators $\omega$ and $D$ are diagonal with respect to a product-state basis of $I_{k}^{z}$-eigenstates $\bigotimes_{k}\left|I_{i_{k}} m_{k}\right\rangle$, whereas the term $X$ is purely off-diagonal in this basis, leading to correlations between different nuclei. We neglect corrections to the diagonal part of $H$ of order $\sim A^{2} / N b$, but retain the term of this size in the offdiagonal part $X$. This approximation is justified since, as we will show, the bath correlation time $\tau_{c}$ is much shorter than the time scale where these diagonal corrections become relevant for sufficiently large Zeeman splitting $b \gg A$, where a Born-Markov approximation is valid: $\tau_{c} \sim N / A \ll N b / A^{2}$. In addition, we ignore corrections to $X$ that are smaller by the factors $A_{k} / b \sim A / N b \ll 1$ and $\gamma_{k} \sim 10^{-3}$. Under these approximations, the various terms in Eq. (2) are given by (see also Appendix B):

$$
\begin{aligned}
& \omega \simeq b+h^{z}, D \simeq b \sum_{k} \gamma_{k} I_{k}^{z}, \\
& X \simeq \frac{1}{2} \sum_{k \neq l} \frac{A_{k} A_{l}}{\omega} I_{k}^{-} I_{l}^{+} .
\end{aligned}
$$

\section{MARKOV APPROXIMATION}

For large $b, H_{\mathrm{hf}}$ leads only to incomplete decay of the longitudinal spin $\left\langle S_{z}\right\rangle_{t}, \underline{15}$ However, it is still possible for the transverse spin $\left\langle S_{+}\right\rangle_{t}$ to decay fully $\underline{16}$ through a pure dephasing process, which we now describe in detail. We assume that the electron and nuclear systems are initially unentangled with each other and that the nuclear spin system is prepared in a narrowed state (an eigenstate of the operator $\omega: \omega|n\rangle=\omega_{n}|n\rangle$ ) through a sequence of weak measurements $\stackrel{27,33,34}{=}$ polarization pumping $\stackrel{35}{5}$ frequency focusing under pulsed optical excitation,$\underline{36}$ or by any other means. For these initial conditions, dynamics of the transverse electron spin $\left\langle S_{+}\right\rangle_{t}$ are described by the exact equation of motion: 15

$$
\begin{aligned}
\left\langle\dot{S_{+}}\right\rangle_{t} & =i \omega_{n}\left\langle S_{+}\right\rangle_{t}-i \int_{0}^{t} d t^{\prime} \Sigma\left(t-t^{\prime}\right)\left\langle S_{+}\right\rangle_{t^{\prime}}, \\
\Sigma(t) & =-i \operatorname{Tr} S_{+} \mathrm{L} e^{-i \mathrm{QL} t} \mathrm{QL}|n\rangle\langle n| S_{-} .
\end{aligned}
$$

Here, $\mathrm{L}$ and $\mathrm{Q}$ are superoperators, defined by their action on an arbitrary operator $O: \mathrm{L} O=[H, O], \mathrm{Q} O=$ $\left(1-|n\rangle\langle n| \operatorname{Tr}_{I}\right) O$, where $\operatorname{Tr}_{I}$ indicates a partial trace over the nuclear spin system.

To remove fast oscillations in $\left\langle S_{+}\right\rangle_{t}$ we transform to a rotating frame, in which we define the coherence factor $x_{t}=2 \exp \left[-i\left(\omega_{n}+\Delta \omega\right) t\right]\left\langle S_{+}\right\rangle_{t}$ and associated memory kernel $\tilde{\Sigma}(t)=\exp \left[-i\left(\omega_{n}+\Delta \omega\right) t\right] \Sigma(t)$, with frequency shift determined self-consistently through $\Delta \omega=-\operatorname{Re} \int_{0}^{\infty} d t \tilde{\Sigma}(t)$. Additionally, we change integration variables to $\tau=t-t^{\prime}$. The equation of motion for $x_{t}$ then reads

$$
\dot{x}_{t}=-i \int_{0}^{t} d \tau \tilde{\Sigma}(\tau) x_{t-\tau} .
$$

If $\tilde{\Sigma}(\tau)$ decays to zero sufficiently quickly $\underline{44}$ on the time scale $\tau_{c} \ll T_{2}$, where $T_{2}$ is the decay time of $x_{t}$, we can approximate $x_{t-\tau} \approx x_{t}$ and extend the upper limit on the integral to $t \rightarrow \infty$ (Markov approximation), giving an exponential coherence decay with a small error $\epsilon(t)$ :

$$
x_{t}=\exp \left(-t / T_{2}\right) x_{0}+\epsilon(t), \frac{1}{T_{2}}=-\operatorname{Im} \int_{0}^{\infty} d t \tilde{\Sigma}(t) .
$$

The non-Markovian correction $\epsilon(t)$ can be bounded precisely if $\tilde{\Sigma}(t)$ is known: $:^{37}$

$$
|\epsilon(t)| \leq|\epsilon(t)|_{\max }=2 \int_{0}^{t} d t^{\prime}\left|\int_{t^{\prime}}^{\infty} d t^{\prime \prime} \tilde{\Sigma}\left(t^{\prime \prime}\right)\right| .
$$

Eq. (9) gives a hard bound on the validity of the Markov approximation, and consequently, any corrections to the exponential decay formula. Fig. 1 demonstrates an application of Eqs. (8) and (9) for decay in a homonuclear spin system, which we discuss below.

\section{HOMONUCLEAR SYSTEM}

If only one spin-carrying nuclear isotope is present, $\gamma_{k}=\gamma$, independent of the nuclear site. We then approximate $\Sigma(t)$ to leading order in the perturbation $V=X S^{z}$ (Born approximation, see Appendix C) by expanding Eq. (6) through iteration of the Dyson identity: $e^{-i \mathrm{LQ} t}=e^{-i \mathrm{~L}_{0} \mathrm{Q} t}-i \int_{0}^{t} d t^{\prime} e^{-i \mathrm{~L}_{0} \mathrm{Q}\left(t-t^{\prime}\right)} \mathrm{L}_{V} \mathrm{Q} e^{-i \mathrm{LQ} t^{\prime}}$, where $\mathrm{L}_{V} O=[V, O]$. Higher-order corrections to the Born approximation will be suppressed by the small parameter $A / \omega_{n}: \underline{15}$ Inserting the result into Eq. (8) we find:

$$
\frac{1}{T_{2}}=\operatorname{Re} \int_{0}^{\infty} d t e^{-i \Delta \omega t}\langle X(t) X\rangle ; X(t)=e^{-i \omega t} X e^{i \omega t} .
$$




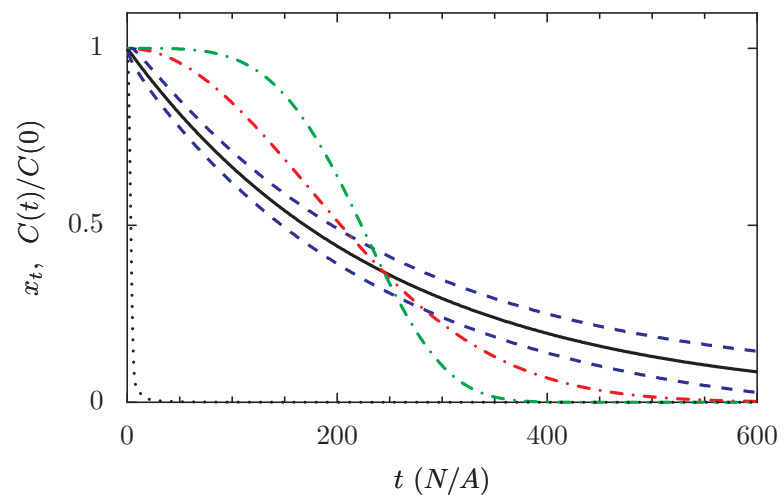

Figure 1: (Color online) Exponential decay $x_{t}=\exp \left(-t / T_{2}\right)$ (solid line) and maximum error bounds $x_{t} \pm|\epsilon(t)|_{\max }$ (dashed lines), found by numerical integration of Eq. (9) with parameters for a two-dimensional quantum dot (before Eq. (14)), $I=3 / 2$ and $A / b=1 / 20$. For comparison, we show the decay curves for super-exponential forms exp $\left[-\left(t / T_{2}\right)^{2}\right]$ and $\exp \left[-\left(t / T_{2}\right)^{4}\right]$ (dot-dashed lines) and rapidly decaying bath correlation function $C(t) / C(0)$ (dotted line, see Eqs. (10) and (11)).

Here, $\langle\cdots\rangle=\langle n|\cdots| n\rangle$ denotes an expectation value with respect to the initial nuclear state. Eq. (10) resembles the standard result for pure dephasing in a weak coupling expansion, where $X(t)$ would represent the bath operator in the interaction picture with an independent bath Hamiltonian. However, for the spin bath there is no such weak coupling expansion, and $X(t)$ appears in the interaction picture with $\omega$, the same operator that provides an effective level splitting for the system. Additionally, the general result for a heteronuclear system including inter-species flip-flops cannot be written in such a compact form $\frac{38}{}$

Previously, it has been shown that a Born-Markov approximation to second order in $V_{\mathrm{ff}}$ leads to no decay: 15 In contrast, a Born-Markov approximation applied to the effective Hamiltonian leads directly to a result that is fourth order in $V_{\mathrm{ff}}$ [Eq. (10)], describing dynamics that become important at times longer than the second-order result. It is not a priori obvious that the effective Hamiltonian, evaluated only to second order in $V_{\mathrm{ff}}$, can be used to accurately calculate rates to fourth order in $V_{\mathrm{ff}}$. We have, however, verified that all results we present here are equivalent to a direct calculation expanded to fourth order in $V_{\text {ff }}$ at leading order in $A / b \ll 1.38$

If the initial nuclear polarization is smooth on the scale of the electron wave function, the matrix elements of operators like $I_{k}^{ \pm} I_{k}^{\mp}$ can be replaced by average values. Neglecting corrections that are small in $A / N b \ll 1$, this gives (see also Appendix D):

$$
C(t)=\langle X(t) X(0)\rangle=\frac{c_{+} c_{-}}{4 \omega_{n}^{2}} \sum_{k \neq l} A_{k}^{2} A_{l}^{2} e^{-i\left(A_{k}-A_{l}\right) t} .
$$

Above, we have introduced the coefficients $c_{ \pm}=I(I+$ $1)-\langle\langle m(m \pm 1)\rangle\rangle$ and the double angle bracket indicates

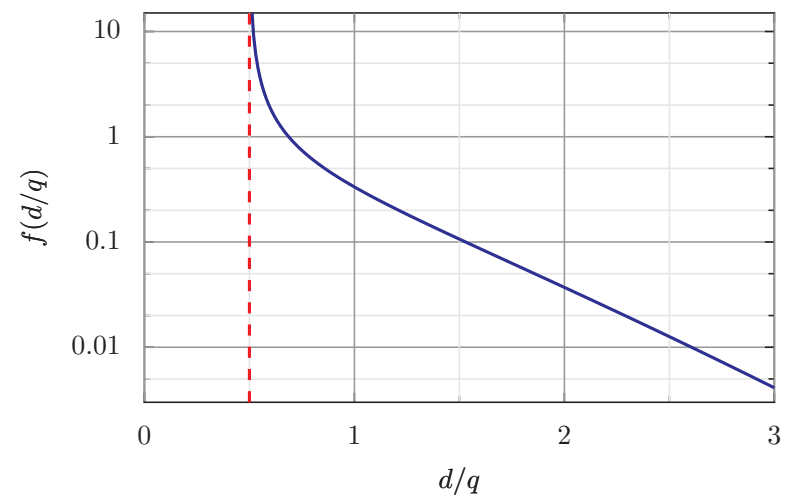

Figure 2: (Color online) Geometrical factor $f(d / q)$ from Eq. (13), where $d=1,2,3$ is the dimension and $q$ characterizes the electron envelope function $\psi(r)=\psi(0) \exp \left[-\left(r / r_{0}\right)^{q} / 2\right]$.

an average over $I_{k}^{z}$ eigenvalues $m \underbrace{15}$

In the limit $N \gg 1$ we can include the term $k=l$ in Eq. (11) and perform the continuum limit $\Sigma_{k} \rightarrow \int d k$ with small corrections. For an isotropic electron wave function of the form $\psi(r)=\psi(0) e^{-\left(r / r_{0}\right)^{q} / 2}$ containing $N$ nuclei within radius $r_{0}$ in $d$ dimensions, the hyperfine coupling constants are distributed according to $A_{k}=A_{0} \exp \left[-(k / N)^{q / d}\right]$, where $k$ is a non-negative index, and we choose $A_{0}$ to normalize $A_{k}$ according to $A=\int_{0}^{\infty} d k A_{k}{ }^{15}$ (see also Appendix $\mathrm{A}$ ).

After performing the continuum limit, $C(t)$ will decay, with characteristic time $\tau_{c}$ given by the inverse bandwidth of nuclear flip-flop excitations $\tau_{c} \sim 1 / A_{0} \sim N / A$. For large $b, 1 / T_{2}$ will be suppressed due to the smallness of $X$ (see Eq. (4) ), whereas $\tau_{c}$ remains fixed. At sufficiently large $b$, it will therefore be possible to reach the Markovian regime, where $\tau_{c}$ is short compared to $T_{2}$ : $\tau_{c} / T_{2} \ll 1$. Evaluating the time integral in Eq. (10), we find the general result to leading order in $A / \omega_{n}$ (see Appendix D):

$$
\begin{aligned}
\frac{1}{T_{2}} & =\frac{\pi}{4} c_{+} c_{-} f\left(\frac{d}{q}\right)\left(\frac{A}{\omega_{n}}\right)^{2} \frac{A}{N}, \\
f(r) & =\frac{1}{r}\left(\frac{1}{3}\right)^{2 r-1} \frac{\Gamma(2 r-1)}{[\Gamma(r)]^{3}}, r>1 / 2 .
\end{aligned}
$$

In Eq. (12), $A / N$ sets the scale for the maximum decay rate in the perturbative regime, the coefficients $c_{ \pm}$set the dependence on the initial nuclear polarization $p(e . g$, with $I=1 / 2$, we have $\left.c_{+} c_{-}=\left(1-p^{2}\right) / 4\right), A / \omega_{n}<1$ gives the small parameter which controls the Born approximation, and $f(d / q)$ is a geometrical factor (plotted in Fig. 2). $f(d / q)$ is exponentially suppressed for $d / q>1$ $\left(f(r) \propto(1 / 3)^{2 r-1}(1 / r)^{r}, r>1\right)$, but $f(d / q) \rightarrow \infty$ for $d / q-1 / 2 \rightarrow 0^{+}$. Due to this divergence, no Markov approximation is possible (within the Born approximation) for $d / q \leq 1 / 2$. We understand the divergence in $f(d / q)$ explicitly from the asymptotic dependence of $C(t)$ at long times: $C(t) \propto 1 / t^{2 d / q}, t \gg N / A, d / q<2 .{ }^{44}$ Surprisingly, there is a difference of nearly two orders of magnitude in $1 / T_{2}$ going from a two-dimensional (2D) 


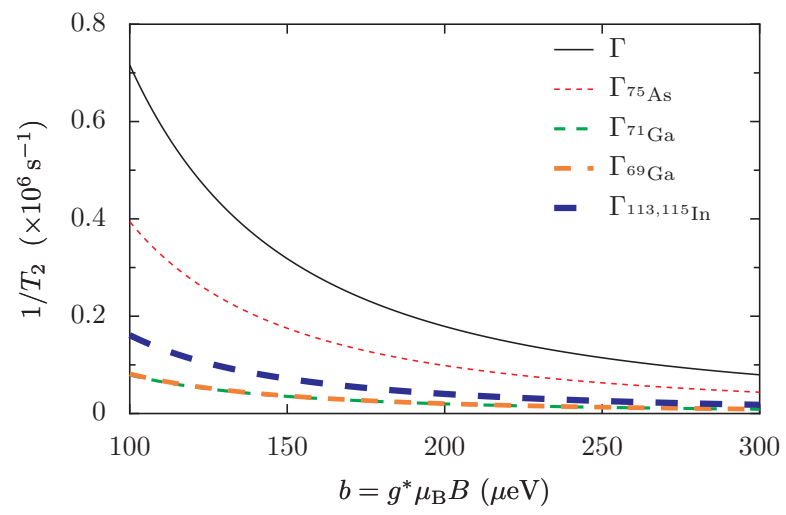

Figure 3: (Color online) Decay rates for an $\operatorname{In}_{x} \mathrm{Ga}_{1-x} \mathrm{As}$ quantum dot with In doping $x=0.05$. Here, we have assumed $N=10^{5}$ and used values of $\nu_{i}$ and $A^{i}$ for GaAs from Ref. [41]: $A^{75} \mathrm{As}=86 \mu \mathrm{eV}, A^{69} \mathrm{Ga}=74 \mu \mathrm{eV}, A^{71} \mathrm{Ga}=$ $96 \mu \mathrm{eV}, \nu_{75} \mathrm{As}=0.5, \nu_{69} \mathrm{Ga}=0.3(1-x), \nu_{71} \mathrm{Ga}=0.2(1-x)$. The hyperfine coupling for In in InAs was taken from Ref. [42]: $A^{113}$ In $\approx A^{115}$ In $\approx A^{\text {In }}=170 \mu e V, \nu_{\text {In }}=x / 2$.

quantum dot with Gaussian envelope function $(d=2$, $q=2, d / q=1)$ to a donor impurity with a hydrogen-like exponential wave function $(d=3, q=1, d / q=3)$, if all other parameters are fixed (see Fig. 2).

We now specialize to an initial uniform unpolarized spin bath, which is nevertheless narrowed: $\omega|n\rangle=b|n\rangle$, with equal populations of all nuclear Zeeman levels (i.e., $\langle\langle m\rangle\rangle=0$ and $\left.\left\langle\left\langle m^{2}\right\rangle\right\rangle=\frac{1}{3} I(I+1)\right)$. For a $2 \mathrm{D}$ quantum dot with a Gaussian envelope function $(d=q=2)$ we find, from Eqs. (12) and (13):

$$
\frac{1}{T_{2}}=\frac{\pi}{3}\left(\frac{I(I+1) A}{3 b}\right)^{2} \frac{A}{N} .
$$

There are two remarkable features of this surprisingly simple result. First, the condition for the validity of the Markov approximation, $T_{2}>\tau_{c} \sim N / A$ will be satisfied whenever $A / b<1$, which is the same condition that validates a Born approximation. Second, $1 / T_{2}$ has a very strong dependence on the nuclear spin $\left(1 / T_{2} \propto I^{4}\right)$. Thus, systems with large-spin nuclei such as In $\left(I_{\text {In }}=9 / 2\right)$ will show relatively significantly faster decay (see, e.g., Fig. 3).

\section{HETERONUCLEAR SYSTEM}

For sufficiently large $b\left(\left|\gamma_{k}-\gamma_{k^{\prime}}\right| b \gg\left|A_{k}-A_{k^{\prime}}\right| \sim\right.$ $A / N)$, heteronuclear flip-flops between two isotopic species with different $\gamma_{k}$ are forbidden due to energy conservation. In this case, $1 / T_{2}$ is given in terms of an incoherent sum, $1 / T_{2}=\Gamma=\sum_{i} \Gamma_{i}$, where $\Gamma_{i}$ is the contribution from flip-flops between nuclei of the common species $i$. Assuming a uniform distribution of all isotopes in a 2D quantum dot with a Gaussian envelope function, we find (see also Appendix D):

$$
\Gamma_{i}=\frac{1}{T_{2}^{i}}=\nu_{i}^{2} \frac{\pi}{3}\left(\frac{I_{i}\left(I_{i}+1\right) A^{i}}{3 b}\right)^{2} \frac{A^{i}}{N} .
$$

The quadratic dependence on isotopic concentration $\nu_{i}$ is particularly striking. Due to this dependence, electron spins in GaAs, where Ga has two naturally occurring isotopic species, whereas As has only one, will show a decay predominantly due to flip-flops between As spins. This is in spite of the fact that all isotopes in GaAs have the same nuclear spin and nominally similar hyperfine coupling constants (see Fig. 3). Interestingly, we note that the relatively large flip-flop rates for In and As, due to large nuclear spin and isotopic concentration, respectively, may partly explain why only Ga (and not In or As) spins have been seen to contribute to coherent effects in experiments on electron transport through (In/Ga)As quantum dots. 39 The same effect may also explain why polarization appears to be transferred more efficiently from electrons to As (rather than Ga) in GaAs quantum dots. 40

\section{CONCLUSIONS}

We have shown that a single electron spin can exhibit a purely exponential decay for narrowed nuclearspin bath initial conditions and in the presence of a sufficiently large electron Zeeman splitting $b$. This work may be important for implementing existing quantum error correction schemes, which typically assume exponential decay of correlation functions due to a Markovian environment. In the limit of large Zeeman splitting $b>A$, where a Born-Markov approximation is valid, we have found explicit analytical expressions for the decoherence time $T_{2}$, giving explicit dependences on the electron wave function, magnetic field, bath polarization, nuclear spin, and isotopic abundance for a general nuclear spin bath. Moreover, within the Born-Markov approximation, we have found a divergence in the decoherence rate $1 / T_{2}$ for a one-dimensional quantum dot, indicating a breakdown of the Markov approximation in this case.

\section{Acknowledgments}

We thank J. Baugh, D. Klauser, and F. H. L. Koppens for useful discussions. We acknowledge funding from the Swiss NSF, NCCR Nanoscience, JST ICORP, QuantumWorks, and an Ontario PDF (WAC).

\section{Appendix A: CONTINUUM LIMIT}

In this appendix, we describe how the dimensionality $d$ and envelope wave function shape parameter $q$ are defined. For further details on the definition of these quan- 
tities, see Ref. [15]. For a homonuclear spin system, the hyperfine coupling constants are given by

$$
A_{k}=A v_{0}\left|\psi\left(\mathbf{r}_{k}\right)\right|^{2}
$$

where $A$ is the total hyperfine coupling constant, $v_{0}$ is the volume occupied by a single-nucleus unit cell, and $\psi(\mathbf{r})$ is the electron envelope wave function. We assume an isotropic electron envelope:

$$
\psi\left(r_{k}\right)=\psi(0) e^{-\frac{1}{2}\left(\frac{r_{k}}{r_{0}}\right)^{q}}
$$

where $r_{0}$ is the effective Bohr radius, defined as the radial distance enclosing $N$ nuclear spins, and $r_{k}$ is the radial distance enclosing $k$ spins. In $d$ dimensions:

$$
\frac{\operatorname{vol}(k \text { spins })}{\operatorname{vol}(N \text { spins })}=\frac{v_{0} k}{v_{0} N}=\left(\frac{r_{k}}{a_{B}}\right)^{d} .
$$

Inserting Eqs. A3 and (A2) into Eq. (A1):

$$
A_{k}=A_{0} e^{-\left(\frac{k}{N}\right)^{q / d}} .
$$

To determine the $k=0$ coupling $A_{0}$, we enforce the normalization:

$$
\sum_{k} A_{k}=A v_{0} \sum_{k}\left|\psi\left(r_{k}\right)\right|^{2} \approx A \int d^{3} r|\psi(r)|^{2}=A .
$$

This gives

$$
A=A_{0} \int_{0}^{\infty} d k e^{-\left(\frac{k}{N}\right)^{q / d}}
$$

Making the change of variables $u=\left(\frac{k}{N}\right)^{q / d}$, we find immediately

$$
A=A_{0} \frac{d}{q} N \int_{0}^{\infty} d u u^{\frac{d}{q}-1} e^{-u}=A_{0} N \frac{d}{q} \Gamma\left(\frac{d}{q}\right),
$$

which gives the final form for $A_{k}$ :

$$
A_{k}=\frac{A}{N \frac{d}{q} \Gamma\left(\frac{d}{q}\right)} e^{-\left(\frac{k}{N}\right)^{q / d}} .
$$

\section{Appendix B: EFFECTIVE HAMILTONIAN}

In this appendix we give details leading to the derivation of the effective Hamiltonian, described by Eqs. (2), (3), and (4) of the main text. Similar effective Hamiltonians have been derived previously in Refs. 32] and [29], but due to some differences in method and approximation, we give additional details here for the interested reader. We begin from the hyperfine Hamiltonian:

$$
\begin{aligned}
H_{\mathrm{hf}} & =H_{0}+V_{\mathrm{ff}} \\
H_{0} & =\left(b+h^{z}\right) S^{z}+b \sum_{k} \gamma_{k} I_{k}^{z} \\
V_{\mathrm{ff}} & =\frac{1}{2}\left(S_{+} h_{-}+S_{-} h_{+}\right) \\
\mathbf{h} & =\sum_{k} A_{k} \mathbf{I}_{k}
\end{aligned}
$$

To find an effective Hamiltonian that eliminates the flipflop term $V_{\text {ff }}$ at leading order, we apply a unitary transformation:

$$
\bar{H}=e^{S} H_{\mathrm{hf}} e^{-S}
$$

where $S=-S^{\dagger}$ to ensure unitarity. We now expand Eq. (B5) in powers of $S$, retaining terms up to $\mathcal{O}\left(V_{\mathrm{ff}}^{3}\right)$, assuming $S \sim \mathcal{O}\left(V_{\text {ff }}\right)$ :

$$
\begin{aligned}
\bar{H}=H_{0}+V_{\mathrm{ff}}-\left[H_{0}, S\right] & -\left[V_{\mathrm{ff}}, S\right] \\
& +\frac{1}{2}\left[S,\left[S, H_{0}\right]\right]+\mathcal{O}\left(V_{\mathrm{ff}}^{3}\right) .
\end{aligned}
$$

To eliminate $V_{\mathrm{ff}}$ at leading order, we must choose $S$ to satisfy $V_{\mathrm{ff}}-\left[H_{0}, S\right]=0$. The $S$ that satisfies this relation is given by

$$
S=\frac{1}{\mathrm{~L}_{0}} V_{\mathrm{ff}} ; \mathrm{L}_{0} O=\left[H_{0}, O\right]
$$

which is of order $V_{\mathrm{ff}}$, justifying our previous assumption: $S \sim \mathcal{O}\left(V_{\mathrm{ff}}\right)$. Re-inserting Eq. (B7) into Eq. (B6), we find, up to corrections that are third-, or higher-order in $V_{\text {ff }}$ :

$$
\begin{aligned}
\bar{H} & =H+\mathcal{O}\left(V_{\mathrm{ff}}^{3}\right), \\
H & =H_{0}+\frac{1}{2}\left[S, V_{\mathrm{ff}}\right]
\end{aligned}
$$

Directly evaluating Eq. (B7) with $H_{0}$ defined in Eq. (B2) and $V_{\mathrm{ff}}$ defined in Eq. (B3) gives

$$
\begin{aligned}
S=\frac{1}{2} \sum_{k} A_{k}( & \frac{1}{b+h^{z}+\frac{A_{k}}{2}-b \gamma_{k}} S^{+} I_{k}^{-} \\
& \left.\quad-\frac{1}{b+h^{z}-\frac{A_{k}}{2}-b \gamma_{k}} S^{-} I_{k}^{+}\right) .
\end{aligned}
$$

Inserting Eq. (B10) into Eq. (B9) gives

$$
\begin{aligned}
H & =|\uparrow\rangle\left\langle\uparrow\left|H_{\uparrow}+\right| \downarrow\right\rangle\langle\downarrow| H_{\downarrow}, \\
H_{\uparrow} & =\frac{1}{2}\left(b+h^{z}\right)+b \sum_{k} \gamma_{k} I_{k}^{z}+h_{\uparrow}, \\
H_{\downarrow} & =-\frac{1}{2}\left(b+h^{z}\right)+b \sum_{k} \gamma_{k} I_{k}^{z}-h_{\downarrow} .
\end{aligned}
$$

Here, the contributions resulting from the term secondorder in $V_{\mathrm{ff}}$ are given explicitly by

$$
\begin{array}{r}
h_{\uparrow}=\frac{1}{8} \sum_{k l} A_{k} A_{l}\left(\frac{1}{b+h^{z}+A_{k} / 2-b \gamma_{k}} I_{k}^{-} I_{l}^{+}\right. \\
\left.+I_{l}^{-} \frac{1}{b+h^{z}-A_{k} / 2-b \gamma_{k}} I_{k}^{+}\right),
\end{array}
$$




$$
\begin{aligned}
h_{\downarrow}=\frac{1}{8} \sum_{k l} A_{k} & A_{l}\left(\frac{1}{b+h^{z}-A_{k} / 2-b \gamma_{k}} I_{k}^{+} I_{l}^{-}\right. \\
+ & \left.I_{l}^{+} \frac{1}{b+h^{z}+A_{k} / 2-b \gamma_{k}} I_{k}^{-}\right) .
\end{aligned}
$$

We can rewrite $H$ in terms of spin operators using $|\uparrow\rangle\langle\uparrow|=\frac{1}{2}+S^{z}$ and $|\downarrow\rangle\langle\downarrow|=\frac{1}{2}-S^{z}$, which gives Eq. (2) from the main text:

$$
\begin{aligned}
H & =(\omega+X) S^{z}+D, \\
X & =\left(1-\mathrm{P}_{\mathrm{d}}\right)\left(h_{\uparrow}+h_{\downarrow}\right), \\
D & =b \sum_{k} \gamma_{k} I_{k}^{z}+\frac{1}{2}\left(h_{\uparrow}-h_{\downarrow}\right), \\
\omega & =b+h^{z}+\mathrm{P}_{\mathrm{d}}\left(h_{\uparrow}+h_{\downarrow}\right) .
\end{aligned}
$$

In the above expressions, we have introduced the diagonal projection superoperator $\mathrm{P}_{\mathrm{d}} O=\sum_{l}|l\rangle\langle l|\langle l|O| l\rangle$, where the index $l$ runs over all nuclear-spin product states $|l\rangle=\bigotimes_{k}\left|I_{k} m_{k}^{l}\right\rangle$. We now apply the commutation relation $\left[I_{k}^{+}, I_{l}^{-}\right]=2 I_{k}^{z} \delta_{k l}$ and expand the prefactors in Eqs. (B14) and (B15) in terms of the smallness parameter $\frac{A_{k}}{b+h^{z}-b \gamma_{k}} \sim \frac{1}{N} \frac{A}{b} \ll 1$. At leading order in the expansion, we find $h_{\uparrow, \downarrow} \approx h_{\uparrow, \downarrow}^{(0)}$, where

$$
\begin{aligned}
h_{\uparrow}^{(0)} & =\frac{1}{8} \sum_{k l} \frac{A_{k} A_{l}}{b+h^{z}-b \gamma_{k}}\left(I_{k}^{-} I_{l}^{+}+I_{l}^{-} I_{k}^{+}\right), \\
h_{\downarrow}^{(0)} & =\frac{1}{8} \sum_{k l} \frac{A_{k} A_{l}}{b+h^{z}-b \gamma_{k}}\left(I_{k}^{+} I_{l}^{-}+I_{l}^{+} I_{k}^{-}\right) .
\end{aligned}
$$

By commuting the nuclear spin operators, Eqs. (B20) and (B21) can be rewritten to give

$$
h_{\downarrow}^{(0)}=h_{\uparrow}^{(0)}+\frac{1}{2} \sum_{k} \frac{A_{k}^{2}}{b+h^{z}-b \gamma_{k}} I_{k}^{z} .
$$

This relation allows us to approximate the various terms in Eqs. (B17), (B18), and (B19):

$$
\begin{aligned}
X & \approx\left(1-\mathrm{P}_{\mathrm{d}}\right)\left(2 h_{\uparrow}^{(0)}\right) \\
& =\frac{1}{4} \sum_{k \neq l} \frac{A_{k} A_{l}}{b+h^{z}-b \gamma_{k}}\left(I_{k}^{-} I_{l}^{+}+I_{l}^{-} I_{k}^{+}\right),
\end{aligned}
$$

and

$$
\begin{gathered}
D \approx \sum_{k}\left(b \gamma_{k}-\frac{A_{k}^{2}}{4\left(b+h^{z}-b \gamma_{k}\right)}\right) I_{k}^{z}, \\
\omega \approx b+h^{z} \\
\quad+\mathrm{P}_{\mathrm{d}}\left(2 h_{\uparrow}^{(0)}\right)+\frac{1}{2} \sum_{k} \frac{A_{k}^{2}}{b+h^{z}-b \gamma_{k}} I_{k}^{z},
\end{gathered}
$$

$$
\begin{aligned}
\omega & \approx b+h^{z} \\
& +\frac{1}{2} \sum_{k} \frac{A_{k}^{2}}{b+h^{z}-b \gamma_{k}}\left(I_{k}\left(I_{k}+1\right)-\left(I_{k}^{z}\right)^{2}\right) .
\end{aligned}
$$

Neglecting further corrections that are smaller by the factor $b \gamma_{k} / \omega \sim \gamma_{k} \sim 10^{-3}$ in Eq. (B23) and terms of order $\lesssim \sum_{k} \frac{A_{k}^{2}}{b+h^{z}-b \gamma_{k}} \sim \frac{A^{2}}{N b}$ in Eqs. (B24) and (B26), we arrive immediately at Eqs. (3) and (4) of the main text. The terms of order $\sim A^{2} / N b$ may become important on a time scale $\tau \sim N b / A^{2}$. In our treatment, this time scale is long compared to the bath correlation time $\tau_{c} \sim N / A$ in the perturbative regime $A / b<1$, and so neglecting these terms is justified.

\section{Appendix C: BORN APPROXIMATION}

In this appendix we give further detail on the Born approximation. We begin from the equation of motion for the transverse spin in the rotating frame $x_{t}$ after applying the Markov approximation, neglecting the correction $\epsilon(t)$ (following Eq. (7)):

$$
\begin{aligned}
\dot{x}_{t} & =-i \int_{0}^{\infty} d \tau \tilde{\Sigma}(\tau) x_{t}, \\
\tilde{\Sigma}(t) & =e^{-i\left(\omega_{n}+\Delta \omega\right) t} \Sigma(t), \\
\Sigma(t) & =-i \operatorname{Tr} S_{+} \mathrm{LQQ} e^{-i \mathrm{LQ} t} \mathrm{LQ}|n\rangle\langle n| S_{-} .
\end{aligned}
$$

In general, it is not simple to find the exact form of the self energy (memory kernel) $\Sigma(t)$. Fortunately, it is possible to generate a systematic expansion in the perturbation $V=X S^{z} \propto 1 / b$, valid for sufficiently large Zeeman splitting $b>A: \underline{15}$

$$
\Sigma(t)=\Sigma^{(2)}(t)+\Sigma^{(4)}(t)+\cdots,
$$

where $\Sigma^{(n)}(t)$ indicates a term of order $\sim \mathcal{O}\left(V^{n}\right) \sim$ $\mathcal{O}\left[\left(\frac{A}{b}\right)^{n}\right]$. The expansion is performed most conveniently in terms of the Laplace-transformed variable

$$
\Sigma(s)=\mathcal{L}[\Sigma(t)]=\int_{0}^{\infty} d t e^{-s t} \Sigma(t) .
$$

We expand the propagator $\mathcal{L}\left[e^{-i \mathrm{LQ} t}\right]=\frac{1}{s+i \mathrm{LQ}}$ by dividing the full Liouvillian into unperturbed and perturbed parts: $\mathrm{L}=\mathrm{L}_{0}+\mathrm{L}_{V}$, where $\mathrm{L}_{0}$ and $\mathrm{L}_{V}$ are defined by their action on an arbitrary operator $O$ through $\mathrm{L}_{0} O=\left[H_{0}, O\right]$ and $\mathrm{L}_{V} O=[V, O]$. To obtain an expansion in terms of the perturbation $\mathrm{L}_{V}$, we now iterate the Dyson identity in Laplace space:

$$
\frac{1}{s+i \mathrm{LQ}}=\frac{1}{s+i \mathrm{~L}_{0} \mathrm{Q}}-i \frac{1}{s+i \mathrm{~L}_{0} \mathrm{Q}} \mathrm{L}_{V} \mathrm{Q} \frac{1}{s+i \mathrm{~L}_{0} \mathrm{Q}}+\mathcal{O}\left(\mathrm{L}_{V}^{2}\right) \text {. }
$$


Inserting the iterated expression (Eq. (C6)) into the Laplace-transformed version of Eq. (C3), we find the self energy in Born approximation (to second order in $V$ ) is

$$
\begin{aligned}
\Sigma^{(2)}(s)=-i \operatorname{Tr}\left[S_{+}\right. & \left(1-i \mathrm{~L}_{0} \mathrm{Q} \frac{1}{s+i \mathrm{~L}_{0}}\right) \\
& \left.\times \mathrm{L}_{V} \frac{1}{s+i \mathrm{~L}_{0}} \mathrm{~L}_{V}|n\rangle\langle n| S_{-}\right] .
\end{aligned}
$$

We have simplified the above expression using the following identities for the projection superoperators $Q=$ $1-|n\rangle\langle n| \operatorname{Tr}_{I}$ and $\mathrm{P}=1-\mathrm{Q}$ :

$$
\begin{aligned}
& \mathrm{PL}_{0} \mathrm{P}=\mathrm{L}_{0} \mathrm{P}, \\
& \mathrm{PL}_{V}|n\rangle\langle n|=0 \text {, } \\
& \mathrm{QL}_{0} \mathrm{Q}=\mathrm{QL}_{0} \text {, }
\end{aligned}
$$

which can be proven directly. To further reduce the above expression, we evaluate the action of $\mathrm{L}_{0}$ and $\mathrm{L}_{V}$ on the electron spin operator $S_{-}$:

$$
\begin{aligned}
\mathrm{L}_{V} S_{-} & =-\frac{1}{2} \mathrm{~L}_{X}^{+} S_{-} \\
\mathrm{L}_{0} S_{-} & =\left(-\frac{1}{2} \mathrm{~L}_{\omega}^{+}+\mathrm{L}_{D}\right) S_{-},
\end{aligned}
$$

where

$$
\begin{aligned}
\mathrm{L}_{X}^{+} O & =[X, O]_{+}, \\
L_{\omega}^{+} O & =[\omega, O]_{+}, \\
L_{D} O & =[D, O],
\end{aligned}
$$

and here we denote anticommutators with a ' + ' subscript: $[A, B]_{+}=A B+B A$. This leads to

$$
\begin{aligned}
\Sigma^{(2)}(s)=- & \frac{i}{4} \operatorname{Tr}_{I}\left[\left(1+\frac{i}{2} \mathrm{~L}_{\omega}^{+} \mathrm{Q} \frac{1}{s-\frac{i}{2} \mathrm{~L}_{\omega}^{+}}\right)\right. \\
& \left.\times \mathrm{L}_{X}^{+} \frac{1}{s+i\left(\mathrm{~L}_{D}-\frac{1}{2} \mathrm{~L}_{\omega}^{+}\right)} \mathrm{L}_{X}^{+}|n\rangle\langle n|\right] .
\end{aligned}
$$

Now, noting that

$$
\begin{aligned}
& \mathrm{Q}|n\rangle\langle n|=0, \\
& \mathrm{Q}|k\rangle\langle k|=| k\rangle\langle k|-| n\rangle\langle n|,
\end{aligned}
$$

we can evaluate Eq. (C16) directly, giving

$$
\begin{aligned}
& \Sigma^{(2)}\left(s+i \omega_{n}\right)=-\frac{i}{2} \sum_{k}\left|X_{k n}\right|^{2}\left(\frac{s+\frac{i}{2} \delta \omega_{n k}}{s+i \delta \omega_{n k}}\right) \\
& \times\left(\frac{1}{s+i\left(\delta D_{k n}+\frac{1}{2} \delta \omega_{n k}\right)}+\frac{1}{s-i\left(\delta D_{k n}-\frac{1}{2} \delta \omega_{n k}\right)}\right),
\end{aligned}
$$

where $\delta D_{k n}=D_{k}-D_{n}, \delta \omega_{n k}=\omega_{n}-\omega_{k}$, and $\omega_{k}, D_{k}$ are the eigenvalues associated with eigenstate $|k\rangle: \omega|k\rangle=$ $\omega_{k}|k\rangle, D|k\rangle=D_{k}|k\rangle$. Additionally, we have denoted $X_{k n}=\langle k|X| n\rangle$.

From Eqs. (C1), (C2), and (C5), the electron-spin decoherence rate within a Born-Markov approximation will now be given by

$$
\frac{1}{T_{2}}=-\operatorname{Im} \Sigma^{(2)}\left(s=i\left(\omega_{n}+\Delta \omega\right)+0^{+}\right),
$$

where $0^{+}$denotes a positive infinitesimal. Our goal here is to find the leading-order dependence of $1 / T_{2}$ on $1 / b$ for large Zeeman splitting: $b>A$. We therefore set $\Delta \omega=$ $-\operatorname{Re} \Sigma^{(2)}\left(s=i\left(\omega_{n}+\Delta \omega\right)+0^{+}\right) \sim \mathcal{O}\left(\frac{A}{N}\left(\frac{A}{b}\right)^{2}\right) \approx 0$, since this term will lead to higher-order corrections in $1 / b$ within the perturbative regime. Additionally, noting that the matrix element $X_{k n}$ induces a flip-flop for spins at two sites $k_{1,2}$, we find $\left|\delta D_{k n}\right|=\left|b\left(\gamma_{k_{1}}-\gamma_{k_{2}}\right)\right|$ and $\left|\delta \omega_{k n}\right|=\left|A_{k_{1}}-A_{k_{2}}\right|$. In the case of a homonuclear system $\gamma_{k_{1}}=\gamma_{k_{2}}$, we can set $\delta D_{k n}=0$ in Eq. (C19). Otherwise, in a sufficiently large magnetic field $\left|b\left(\gamma_{k_{1}}-\gamma_{k_{2}}\right)\right|>\left|A_{k_{1}}-A_{k_{2}}\right|$, we find a negligible contribution to the decoherence rate for terms from two different isotopic species (where $\gamma_{k_{1}} \neq \gamma_{k_{2}}$ ), i.e., heteronuclear flip-flops no longer conserve energy, although homonuclear fip-flops (for which $\gamma_{k_{1}}=\gamma_{k_{2}}$ ) will still occur. Restricting the sum to homonuclear flip-flops and setting $\delta D_{n k}=0$ in this regime gives

$$
\Sigma^{(2)}\left(s+i \omega_{n}\right)=-i \sum_{j} \sum_{k}\left|X_{k n}^{j}\right|^{2} \frac{1}{s+i \delta \omega_{n k}},
$$

where $X_{k n}^{j}=\left\langle k\left|X^{j}\right| n\right\rangle$ and $X^{j}$ is restricted to run over flip-flops between nuclei of the common species $j$ at sites denoted by the indices $k_{j}, l_{j}$ :

$$
X^{j}=\frac{1}{2} \sum_{k_{j} \neq l_{j}} \frac{A_{k_{j}}^{j} A_{l_{j}}^{j}}{\omega} I_{k_{j}}^{-} I_{l_{j}}^{+} .
$$

Inserting Eq. (C21) for a homonuclear system (one isotopic species $j$ ) into Eq. (C20) and inverting the Laplace transform leads directly to Eq. (10) of the main text.

\section{Appendix D: DECOHERENCE RATE}

Applying Eq. (C20) (setting $\Delta \omega \approx 0$ ) with Eq. (C21) gives the rate

$$
\frac{1}{T_{2}}=\pi \sum_{j} \sum_{k}\left|X_{k n}^{j}\right|^{2} \delta\left(\delta \omega_{k n}\right)
$$

which can be found directly from the formula

$$
\frac{1}{x \pm i 0^{+}}=\mathcal{P} \frac{1}{x} \mp i \pi \delta(x),
$$


where $\mathcal{P}$ indicates that the principle value should be taken in any integral over $x$. Rewriting Eq. (D1) using the definition of $X^{j}$ given in Eq. (C22):

$$
\begin{aligned}
\frac{1}{T_{2}} & =\frac{\pi}{4} \\
& \times \sum_{j} \sum_{k_{j} \neq l_{j}} \frac{c_{-}^{j k_{j}} c_{+}^{j l_{j}}}{\omega_{k} \omega_{n}}\left(A_{k_{j}}^{j}\right)^{2}\left(A_{l_{j}}^{j}\right)^{2} \delta\left(A_{k_{j}}^{j}-A_{l_{j}}^{j}\right),
\end{aligned}
$$

where $k_{j}$ and $l_{j}$ are restricted to run over sites occupied by isotopic species $j$. The coefficients $c_{ \pm}^{j k_{j}}$ give the expectation value of the operator $I_{k_{j}}^{\mp} I_{k_{j}}^{ \pm}$with respect to the initial state:

$$
\begin{aligned}
c_{ \pm}^{j k_{j}} & =\left\langle n\left|I_{k_{j}}^{\mp} I_{k_{j}}^{ \pm}\right| n\right\rangle, \\
& =I^{j}\left(I^{j}+1\right)-\left\langle n\left|I_{k_{j}}^{z}\left(I_{k_{j}}^{z} \pm 1\right)\right| n\right\rangle .
\end{aligned}
$$

With small corrections of order $A / N b \ll 1$, we can replace $\omega_{k} \simeq \omega_{n}$ in the denominator of Eq. (D3). If the various nuclear isotopes are uniformly distributed with isotopic concentrations $\nu_{j}$, we allow the sum over $k_{j}, l_{j}$ to extend over all sites $k, l$ at the expense of a weight factor $\nu_{j}$ for each index:

$$
\sum_{k_{j} \neq l_{j}} \approx \nu_{j}^{2} \sum_{k \neq l}
$$

Additionally, we assume that the system is uniformly polarized on the scale of variation of the hyperfine coupling constants so that the coefficients $c_{ \pm}^{j k}$ can be replaced by average values $c_{ \pm}^{j}=\left\langle\left\langle c_{ \pm}^{j k}\right\rangle\right\rangle$ (double angle brackets indicate an average over all sites) and taken out of the sum. Finally, we change the sums over sites to a double integral using the prescription and coupling constants described in Appendix A neglecting the small $\mathcal{O}(1 / N)$ correction due to the requirement $k \neq l$ :

$$
\sum_{k \neq l} \rightarrow \int_{0}^{\infty} d k \int_{0}^{\infty} d l
$$

These approximations give

$$
\begin{aligned}
\frac{1}{T_{2}}=\frac{\pi}{4 \omega_{n}^{2}} \\
\times \sum_{j} \nu_{j}^{2} c_{-}^{j} c_{+}^{j} \int_{0}^{\infty} d k \int_{0}^{\infty} d l\left(A_{k}^{j}\right)^{2}\left(A_{l}^{j}\right)^{2} \delta\left(A_{k}^{j}-A_{l}^{j}\right) .
\end{aligned}
$$

Inserting the coupling constants defined by Eq. (A8) and evaluating the integrals gives

$$
\frac{1}{T_{2}}=\frac{\pi}{4} f\left(\frac{d}{q}\right) \sum_{j} \nu_{j}^{2} c_{-}^{j} c_{+}^{j} \frac{A^{j}}{N}\left(\frac{A^{j}}{\omega_{n}}\right)^{2},
$$

with the geometrical factor $f(d / q)$ given by Eq. (13) of the main text. Eq. (D9) reduces to Eqs. (12), (14), and (15) of the main text in the special cases discussed there.
1 D. Loss and D. P. DiVincenzo, Phys. Rev. A 57, 120 (1998).

2 R. Hanson, L. P. Kouwenhoven, J. R. Petta, S. Tarucha, and L. M. K. Vandersypen, Rev. Mod. Phys. 79, 1217 (2006).

3 M. Leuenberger and D. Loss, Nature 410, 789 (2001).

${ }^{4}$ F. Jelezko, T. Gaebel, I. Popa, A. Gruber, and J. Wrachtrup, Phys. Rev. Lett. 92, 76401 (2004).

${ }^{5}$ R. Vrijen, E. Yablonovitch, K. Wang, H. W. Jiang, A. Balandin, V. Roychowdhury, T. Mor, and D. DiVincenzo, Phys. Rev. A 62, 012306 (2000).

6 J. R. Petta, A. C. Johnson, J. M. Taylor, E. A. Laird, A. Yacoby, M. D. Lukin, C. M. Marcus, M. P. Hanson, and A. C. Gossard, Science 309, 2180 (2005).

7 F. H. L. Koppens, C. Buizert, K. J. Tielrooij, I. T. Vink, K. C. Nowack, T. Meunier, L. P. Kouwenhoven, and L. M. K. Vandersypen, Nature 442, 766 (2006).

8 E. Abe, K. M. Itoh, J. Isoya, and S. Yamasaki, Phys. Rev. B 70, 033204 (2004).

${ }^{9}$ L. Childress, G. Dutt, J. Taylor, A. Zibrov, F. Jelezko, J. Wrachtrup, P. Hemmer, and M. Lukin, Science 314, 281 (2006).

10 R. Hanson, F. M. Mendoza, R. J. Epstein, and D. D. Awschalom, Phys. Rev. Lett. 97, 87601 (2006).

11 A. Ardavan, O. Rival, J. J. L. Morton, S. J. Blundell, A. M.
Tyryshkin, G. A. Timco, and R. E. P. Winpenny, Phys. Rev. Lett. 98, 057201 (2007).

12 F. Troiani (2007), (private communication).

13 A. V. Khaetskii, D. Loss, and L. Glazman, Phys. Rev. Lett. 88, 186802 (2002).

14 R. de Sousa and S. Das Sarma, Phys. Rev. B 68, 115322 (2003).

15 W. A. Coish and D. Loss, Phys. Rev. B 70, 195340 (2004).

16 C. Deng and X. Hu, Phys. Rev. B 73, 241303(R) (2006).

17 W. M. Witzel and S. Das Sarma, Phys. Rev. Lett. 98, 77601 (2007).

18 W. Yao, R. B. Liu, and L. J. Sham, Phys. Rev. Lett. 98, 77602 (2007).

19 F. H. L. Koppens, D. Klauser, W. A. Coish, K. C. Nowack, L. P. Kouwenhoven, D. Loss, and L. M. K. Vandersypen, Phys. Rev. Lett. 99, 106803 (2007).

20 I. A. Merkulov, A. L. Efros, and M. Rosen, Phys. Rev. B 65, 205309 (2002).

21 S. I. Erlingsson and Y. V. Nazarov, Phys. Rev. B 70, 205327 (2004).

22 E. Yuzbashyan, B. Altshuler, V. Kuznetsov, and V. Enolskii, J. Phys. A: Math. Gen. 38, 7831 (2005).

23 K. A. Al-Hassanieh, V. V. Dobrovitski, E. Dagotto, and B. N. Harmon, Phys. Rev. Lett. 97, 37204 (2006).

24 G. Chen, D. L. Bergman, and L. Balents, Phys. Rev. B 
76, 045312 (2007).

25 H. P. Breuer, D. Burgarth, and F. Petruccione, Phys. Rev. B 70, 45323 (2004).

26 B. M. Terhal and G. Burkard, Phys. Rev. A 71, 012336 (2005).

27 D. Klauser, W. A. Coish, and D. Loss, Phys. Rev. B 73, 205302 (2006).

28 A. Abragam, Principles of nuclear magnetism (Oxford University Press (London), 1962), ch. VI, Eqs. (34,35).

${ }^{29}$ W. Yao, R.-B. Liu, and L. J. Sham, Phys. Rev. B 74, 195301 (2006).

30 W. M. Witzel and S. Das Sarma, Phys. Rev. B 74, 035322 (2006).

31 G. Yusa, K. Muraki, K. Takashina, K. Hashimoto, and Y. Hirayama, Nature (London) 434, 1001 (2005).

32 N. Shenvi, R. de Sousa, and K. B. Whaley, Phys. Rev. B 71, 224411 (2005).

${ }^{33}$ G. Giedke, J. M. Taylor, D. D'Alessandro, M. D. Lukin, and A. Imamoğlu, Phys. Rev. A 74, 032316 (2006).

34 D. Stepanenko, G. Burkard, G. Giedke, and A. Imamoglu, Phys. Rev. Lett. 96, 136401 (2006).

35 G. Ramon and X. Hu, Phys. Rev. B 75, 161301(R) (2007).

36 A. Greilich, A. Shabaev, D. R. Yakovlev, A. L. Efros, I. A. Yugova, D. Reuter, A. D. Wieck, and M. Bayer, Science 317, 1896 (2007).
37 E. Fick and G. Sauermann, The Quantum Statistics of Dynamic Processes (Springer-Verlag, Berlin, 1990), eq. (13.1.26).

38 W. A. Coish, J. Fischer, and D. Loss (2007), (in preparation).

39 K. Ono and S. Tarucha, Phys. Rev. Lett. 92, 256803 (2004).

40 S. Foletti, J. Martin, M. Dolev, D. Mahalu, V. Umansky, and A. Yacoby, arXiv:0801.3613v1 [cond-mat.meshall] (2008)

41 D. Paget, G. Lampel, B. Sapoval, and V. I. Safarov, Phys. Rev. B 15, 5780 (1977).

42 R.-B. Liu, W. Yao, and L. J. Sham, New J. Phys. 9, 226 (2007).

43 D. P. DiVincenzo and D. Loss, Phys. Rev. B 71, 035318 (2005).

44 The integral in Eq. (8) becomes undefined if the memory kernel has an asymptotic time dependence $\tilde{\Sigma}(t) \sim 1 / t^{\alpha}$, where $\alpha \leq 1$, and consequently the Markov approximation breaks down in this case. A weaker version of Markovian violation can occur more generally for $\alpha \leq 2$, in which case the bound, Eq. (9), may still be small for times $t \sim T_{2}$, but grows unbounded in time. This situation occurs, for example, in the ohmic spin-boson model. ${ }^{43}$ 